\title{
Braid ordering and the geometry of closed braid
}

\author{
TETSUYA ITO
}

\begin{abstract}
We study the relationships between the Dehornoy ordering of the braid groups and the topology and geometry of the closed braid complements. We show that the Dehornoy floor of braids, which is a nonnegative integer determined by the Dehornoy ordering, tells us the position of essential surfaces in the closed braid complements. Furthermore, we prove that if the Dehornoy floor of a braid is bigger than or equal to two, then the Nielsen-Thurston classification of braids and the geometric structure of the closed braid complements are in one-to-one correspondence.
\end{abstract}

57M25; 57M50

\section{Introduction}

Let $B_{n}$ be the degree $n$ braid group, and $\sigma_{1}, \ldots, \sigma_{n-1}$ be the standard generators of $B_{n}$. We denote by $\hat{\beta}$ the oriented link obtained by closing a braid $\beta$ and call it a closed braid or the closure of $\beta$.

In the 1990's Patrick Dehornoy found a new feature of the braid groups, a leftorderability [9]. The Dehornoy ordering $<_{D}$ of the braid group $B_{n}$ is a left-invariant total ordering defined as follows. For $n$-braids $\alpha$ and $\beta$, we define $\alpha<_{D} \beta$ if the braid $\alpha^{-1} \beta$ admits a word representative which contains no $\sigma_{1}^{ \pm 1}, \sigma_{2}^{ \pm 1}, \ldots, \sigma_{i-1}^{ \pm 1}, \sigma_{i}^{-1}$, but contains at least one $\sigma_{i}$ for some $i$. The fact that this simple definition indeed defines a left-invariant total ordering is highly nontrivial. Today there are many proofs of the fact that this definition indeed defines a left-invariant total ordering (see Dehornoy et al [10]), and the Dehornoy ordering is considered as the natural structure of the braid groups.

The aim of this paper is to study the relationships between the geometry and topology of the closed braid complements and the Dehornoy ordering.

To this end, we introduce the Dehornoy floor of braids, which is a measure of a complexity of braids using the Dehornoy ordering. The notion of the Dehornoy floor that appeared in Malyutin and Netsvetaev [18] and Malyutin [17] is slightly different from the definition given in this paper. 
Definition 1.1 (Dehornoy floor) The Dehornoy floor $[\beta]_{D}$ of an $n$-braid $\beta$ is a nonnegative integer defined by the formula

$$
[\beta]_{D}=\min \left\{m \in \mathbb{Z}_{\geq 0} \mid \Delta^{-2 m-2}<_{D} \beta<_{D} \Delta^{2 m+2}\right\}
$$

where $\Delta=\left(\sigma_{1} \sigma_{2} \cdots \sigma_{n-1}\right)\left(\sigma_{1} \sigma_{2} \cdots \sigma_{n-2}\right) \cdots\left(\sigma_{1} \sigma_{2}\right)\left(\sigma_{1}\right)$ is the Garside fundamental $n$-braid.

The Dehornoy floor is far from a knot invariant. The value of the Dehornoy floor even changes under conjugation of braids. Nevertheless we can extract geometrical information about closed braids via the Dehornoy floor.

Our first main result is the following.

Theorem 1.2 Let $\beta$ be an $n$-braid and $F$ be an oriented essential closed surface of genus $g(\neq 0)$ in the complement of the closed braid $\widehat{\beta}$.

(1) If $F$ is tiled, then $[\beta]_{D}<g+1$.

(2) If $F$ is mixed-foliated, then $[\beta]_{D}<2 g$.

(3) If $[\beta]_{D} \geq 2 g$, then $F$ is circular-foliated.

In the above statements, the notions of tiled, mixed-foliated and circular-foliated are derived from Birman and Menasco's braid foliation theory. These notions represent, roughly speaking, the position of the surface with respect to the axis of the closed braid. The precise definitions of these notions will be given in Section 3, in which we will present a summary of braid foliation theory.

Theorem 1.2 means that the existence of essential surfaces in a closed braid complement restricts the value of the Dehornoy floor. In particular, if the Dehornoy floor is relatively large with respect to the genus of the surface, then the surface can be isotoped so that it does not intersect the axis of the closed braid.

As an application of Theorem 1.2, we give relationships between the Nielsen-Thurston classification of braids and the geometric structure of the closed braid complements. Recall that the Nielsen-Thurston theorem states that elements of the mapping class groups are classified into three types according to their dynamics, periodic, reducible and pseudo-Anosov. On the other hand, Thurston's hyperbolization theorem shows that knots are classified into three types by the geometry of their complements, namely, torus knots, satellite knots and hyperbolic knots.

As is well-known, the Nielsen-Thurston classification deeply involves the geometric structure of 3-dimensional manifolds. Let $F_{\varphi}$ be the mapping torus of a surface 
homomorphism $\varphi: F \rightarrow F$. Then $F_{\varphi}$ is hyperbolic if and only if $\varphi$ is pseudo-Anosov, $F_{\varphi}$ is Seifert-fibered if and only if $\varphi$ is periodic, and $F_{\varphi}$ contains an essential torus (hence we can perform JSJ decomposition) if and only if $\varphi$ is reducible. Thus, there is the one-to-one correspondence between the geometry of the mapping torus and the Nielsen-Thurston classification of its monodromy (see Fathi, Laudenbach and Poenaru [11]).

However, in the braid groups and knots case, although there are many similarities between the Nielsen-Thurston classification and the geometric structure of the closed braid complements, in general there is no such one-to-one correspondence between them. For example, a periodic braid $\sigma_{1}^{3}$, a reducible braid $\sigma_{2} \sigma_{1} \sigma_{3} \sigma_{2} \sigma_{1}$ and a pseudoAnosov braid $\sigma_{1}^{3} \sigma_{2}^{-1}$ represent the same $(2,3)$-torus knot.

Thus, it is an interesting problem to study the relationships between the NielsenThurston classification of braids and the geometric structure of knot complements. In [16], Los showed that these two classifications are related by exchange moves. Theorem 1.3 provides an alternative, but more general partial answer to this problem.

Theorem 1.3 Let $\beta \in B_{n}$ be a braid whose closure is a knot. If $[\beta]_{D} \geq 2$, the following holds.

(1) $\beta$ is periodic if and only if $\hat{\beta}$ is a torus knot.

(2) $\beta$ is reducible if and only if $\hat{\beta}$ is a satellite knot.

(3) $\beta$ is pseudo-Anosov if and only if $\hat{\beta}$ is a hyperbolic knot.

This theorem implies that under the condition $[\beta]_{D} \geq 2$, the Nielsen-Thurston classification of braids and the geometry of their closures are in one-to-one correspondence. The condition $[\beta]_{D} \geq 2$ in Theorem 1.3 is the best-possible. The pseudo-Anosov 3 -braid $\beta=\sigma_{1} \sigma_{2}^{3} \sigma_{1}^{2}$ has Dehornoy floor 1 , but its closure $\hat{\beta}$ is a connected sum of two trefoils, so it is a satellite knot.

The plan of this paper is the following. In Section 2, we study properties of the Dehornoy floor and provide a connection between the Dehornoy floor and the blockstrand diagrams. Section 3 provides a brief exposition of Birman and Menasco's braid foliation theory which will be used later. The proof of Theorem 1.2 will be given in Section 4. Section 5 is devoted to the proof of Theorem 1.3 and provides some applications. 
Acknowledgments The author would like to thank his adviser Toshitake Kohno for helpful suggestions and advices for preparing this manuscript and Eiko Kin for useful conversations. He gratefully thanks the anonymous referee for providing the wonderful point of view for the proof of Lemma 4.2. Finally, he wishes to express his gratitude to Joan Birman, whose comments made the paper more readable. This research was supported by JSPS Research Fellowships for Young Scientists.

\section{Braid ordering and Dehornoy floor, block-strand diagrams}

In this section we study fundamental properties of the Dehornoy ordering and the Dehornoy floor, and provide a connection between the Dehornoy floor and block-strand diagram description of braids. See Dehornoy et al [10] for the basics of the braid orderings.

\subsection{Dehornoy orderings}

We review the geometrical definition of the Dehornoy ordering given by Fenn et al [12], which will be used later.

Let $D_{n}=\{z \in \mathbb{C}|| z \mid<n+1\}-\{1,2, \ldots, n\}$ be the $n$-punctured disc and $\Gamma=$ $\left\{z \in D_{n} \mid \operatorname{Im}(z)=0\right\}$ be the diameter of $D_{n}$ which is oriented from left to right. The braid group $B_{n}$ is identified with the mapping class group $\operatorname{MCG}\left(D_{n}, \partial D_{n}\right)$, which is the group of isotopy classes of homeomorphisms of $D_{n}$ fixing the boundary $\partial D_{n}$ pointwise. The standard generator $\sigma_{i}$ corresponds to the isotopy class of the half Dehntwist along the segment of $\Gamma$ which connects the $i$-th and the $(i+1)-$ st punctures. Throughout this paper we assume $B_{n}$ acts on $D_{n}$ from left.

For $n$-braids $\alpha$ and $\beta$, consider the image of $\Gamma, \alpha(\Gamma)$ and $\beta(\Gamma)$. By taking appropriate representative homeomorphisms, we can assume that $\alpha(\Gamma)$ and $\beta(\Gamma)$ have minimal intersections. If $\alpha \neq \beta$, then the images $\alpha(\Gamma)$ and $\beta(\Gamma)$ must diverge at some puncture point. Then $\alpha{ }_{D} \beta$ is equivalent to the condition that the $\operatorname{arc} \beta(\Gamma)$ moves the left side of $\alpha(\Gamma)$ at the first divergence point [12]. Thus, the Dehornoy floor can be seen as the absolute value of the winding number of first small initial segment of $\Gamma$.

The Dehornoy ordering has the following property, called Property $S$ or the Subword property [10].

Proposition 2.1 (Property $S$ ) For braids $\beta_{1}, \beta_{2} \in B_{n}$ and $1 \leq i \leq n-1$,

$$
\beta_{1} \sigma_{i} \beta_{2}>_{D} \beta_{1} \beta_{2}>_{D} \beta_{1} \sigma_{i}^{-1} \beta_{2}
$$

holds. 
The property $S$ implies that we can insert (resp. delete) generator $\sigma_{i}$ into a braid word without decreasing (resp. increasing) the Dehornoy ordering. This property will be used to establish an estimation of the Dehornoy floor in next section.

\subsection{Estimation of the Dehornoy floor}

In this section we study properties of the Dehornoy floor.

First of all, we remark that when we use the Dehornoy floor, which $B_{n}$ a braid $\beta$ belongs to is very important. For example, let $\beta=\left(\sigma_{1} \sigma_{2}\right)^{3 k+1}(k \geq 0)$ be a braid word. If we consider $\beta$ as a 3-braid, then $[\beta]_{D}=k$. On the other hand, if we regard $\beta$ as a 4-braid, then $[\beta]_{D}=0$. Fortunately, if we use the braid groups to describe links, the number of strands is always implicit, so there is little possibility for confusion about the number of braid strands.

We also remark that as we mentioned earlier, the Dehornoy floor is not a conjugacy invariant of braids. For example, a 3-braid $\Delta^{2} \sigma_{1} \sigma_{2}^{-1}$ has the Dehornoy floor one, but its conjugate $\Delta\left(\Delta^{2} \sigma_{1} \sigma_{2}^{-1}\right) \Delta^{-1}=\Delta^{2} \sigma_{2} \sigma_{1}^{-1}$ has the Dehornoy floor zero.

As a first step, we summarize the basic properties of the Dehornoy floor proved by Malyutin and Netsvetaev [18] and Malyutin [17].

Proposition $2.2[18 ; 17]$ Let $\alpha, \beta$ be $n$-braids.

(1) If the braid $\beta$ admits a word representative which contains $s(>0)$ occurrence of $\sigma_{1}$ and $k(>0)$ occurrence of $\sigma_{1}^{-1}$, then $[\beta]_{D}<\max \{s, k\}$.

(2) $\left|[\beta]_{D}-[\alpha]_{D}\right| \leq 1$ if $\beta$ and $\alpha$ are conjugate.

(3) $[\alpha \beta]_{D} \leq[\alpha]_{D}+[\beta]_{D}+1$ holds.

These properties of the Dehornoy floor are direct consequences of the facts that $\Delta^{2}$ is central in $B_{n}$ and that $\sigma_{i}^{-1} \Delta^{2}$ is a positive braid for all $i$.

Now we present two new estimation results about the Dehornoy floor. First we sharpen the estimation of the Dehornoy floor given in Proposition 2.2 (1).

Proposition 2.3 If a braid $\beta$ is conjugate to a braid $\alpha$ which admits a word representative with $s(>0)$ occurrences of $\sigma_{1}$ and $k(>0)$ occurrences of $\sigma_{1}^{-1}$, then $[\beta]_{D}<\max \{s, k\}$. 
Proof We show $\beta{ }_{{ }_{D}} \Delta^{2 k}$. The proof of $\Delta^{-2 s}{ }_{{ }_{D}} \beta$ is similar. The main idea of the proof is simple. We insert a generator $\sigma_{i}$ into the braid $\beta$ so that the action of $\beta$ on the punctured disc $D_{n}$ is simple and easy to compare with $\Delta^{2 k}$. Since inserting generators does not decrease the Dehornoy ordering, one can prove $\beta{ }_{D} \Delta^{2 k}$ by comparing the modified braid and $\Delta^{2 k}$.

From the assumption, the braid $\beta$ is written as

$$
\beta=\gamma^{-1}\left(W_{0} \sigma_{1} W_{1} \sigma_{1} \cdots W_{k}\right) \gamma
$$

where $\gamma \in B_{n}$ and $W_{i}$ is a braid word which contains no $\sigma_{1}$. By putting $\gamma^{*}=W_{0}^{-1} \gamma$, we delete $W_{0}$ and write $\beta$ as

$$
\beta=\gamma^{*-1}\left(\sigma_{1} W_{1} \cdots \sigma_{1}\left(W_{k} W_{0}\right)\right) \gamma^{*} .
$$

Let $D=\left(\sigma_{2} \sigma_{3} \cdots \sigma_{n-1}\right)^{n-1}$. By inserting positive generators $\sigma_{1}, \ldots, \sigma_{n-1}$ into the words $W_{i}$, we can obtain the word $\bar{\beta}$, which has the form

$$
\bar{\beta}=\gamma^{*-1}\left(\sigma_{1} D^{p}\right)^{k} \gamma^{*} \text {. }
$$

From Property $S$ of the Dehornoy ordering, $\bar{\beta}>_{D} \beta$ holds.

Now we show $\bar{\beta}<_{D} \Delta^{2 k}$, which is equivalent to $\left(\sigma_{1} D^{p}\right)^{k} \gamma^{*}<_{D} \Delta^{2 k} \gamma^{*}$. First we consider the case $k=1$. Let $C$ be a circle in $D_{n}$ enclosing all but the first puncture point. Then the braid $D$, viewing as an element of $\operatorname{MCG}\left(D_{n}, \partial D_{n}\right)$, corresponds to the Dehn-twists along $C$. Similarly, the braid $\Delta^{2}$ corresponds to the Dehn-twist along the boundary $\partial D_{n}$. Thus by calculating the image of the horizontal diameter $\Gamma$, we conclude that the image of $\Delta^{2} \gamma^{*}(\Gamma)$ moves left side of $\sigma_{1} D^{p} \gamma^{*}(\Gamma)$ for all $p$, thus $\sigma_{1} D^{p} \gamma^{*}{ }_{D} \Delta^{2} \gamma^{*}$ holds. Induction on $k$ gives the desired result.

Proposition 2.3 is a key to the connection between braid ordering and the geometric properties of braids, which will be described in the next section.

Next we provide another estimation result of the Dehornoy floor which uses the band generators. This estimation is used in Ito [14] to relate the Dehornoy floor and knot genus. For $1 \leq i<j \leq n$, let $a_{i, j}$ be an $n$-braid

$$
a_{i, j}=\left(\sigma_{j} \sigma_{j-1} \cdots \sigma_{i+1}\right) \sigma_{i}\left(\sigma_{i+1}^{-1} \cdots \sigma_{j}^{-1}\right) .
$$

The braids $\left\{a_{i, j}\right\}$ are called band generators. This name derived from the fact that the band generator $a_{i, j}$ can be seen as a boundary of twisted band which connects the $i$-th and $j$-th strands. The band generators determines a Garside structure on the braid group $B_{n}$ as do the standard generators $\left\{\sigma_{i}\right\}$. We do not describe these properties of band generator because we do not use them. See Birman, Ko and Lee [3] for details. 
Proposition 2.4 If an $n$-braid $\beta$ is conjugate to a braid $\beta^{\prime}$ which is represented by the product of $m$ band generators, then

$$
\frac{m+1}{n}>[\beta]_{D}
$$

holds.

Proof Let $\beta=\gamma^{-1} W \gamma$ where $W$ is a product of $m$ band generators and $\gamma \in B_{n}$. We show $\beta<_{D} \Delta^{2 p}$, where $p$ is the largest nonnegative integer which is less than or equal to $(m+1) / n$. The proof of $\Delta^{-2 p}<_{D} \beta$ is similar. It suffices to consider the case when $W$ is a product of positive band generators, because we can delete the inverse of band generators without decreasing the Dehornoy ordering by Property $S$.

First we consider the case $m=n-1$. Let $G$ be a graph in $D_{n}$, whose vertices are puncture points of $D_{n}$ and whose edges are arcs connecting two distinct vertices, lying entirely in the upper half of the disc $D_{n}$. We denote by $e_{i, j}$ the edge of $G$ connecting the $i$-th and $j$-th vertices. Let $D^{\prime}$ be a subdisc of $D_{n}$ which is bounded by the edge-path $e_{1,2} \cup e_{2,3} \cup \cdots \cup e_{n-1, n} \cup e_{n, 1}$. The band generator $a_{i, j}$ corresponds to the isotopy class of the half Dehn-twist along the edge $e_{i, j}$, and the braid $\gamma^{-1} a_{i, j} \gamma$ corresponds to the isotopy class of the half Dehn-twist along the arc $\gamma\left(e_{i, j}\right)$. The square of the Garside fundamental braid $\Delta^{2}$ corresponds to the Dehn-twist along $\partial D^{\prime}$. Since $\partial D^{\prime}$ consists of $n$ edges, so does $\partial \gamma\left(D^{\prime}\right)$. Let $e_{1}^{\prime}, e_{2}^{\prime}, \ldots, e_{n}^{\prime}$ be edges of $\gamma(G)$ such that edge-path $e_{1}^{\prime} \cup e_{2}^{\prime} \cup \cdots \cup e_{n}^{\prime}$ forms the boundary $\partial \gamma\left(D^{\prime}\right)$. We choose $e_{1}^{\prime}$ as the edge which intersects the horizontal diameter $\Gamma$ at the leftmost point. That is, we choose the edge $e_{1}^{\prime}$ so that the first intersection point of $\gamma\left(\partial D^{\prime}\right)$ with $\Gamma$ lies on $e_{1}^{\prime}$. Then the maximal element of the product of $n-1$ half Dehn-twists along the arcs $\left\{\gamma\left(e_{i, j}\right)\right\}$ is $b_{n-1} \cdots b_{2} b_{1}$ where $b_{i}$ is a half Dehn-twist along the edge $e_{i}^{\prime}$. On the other hand, by seeing the image of $\Gamma$, we obtain that the braid $b_{n-1} \cdots b_{2} b_{1}$ is strictly smaller than the Dehn-twist along $\partial \gamma\left(D^{\prime}\right)$ that is, $\Delta^{2}$. Therefore we conclude $\beta<_{D} \Delta^{2}$. By iterating this argument, we obtain the desired result.

Finally we study the complexity issue of the calculation of the Dehornoy floor. There are many algorithms to compare given braids. One of the most efficient and practical algorithm uses the band generators and the normal form of braids called the rotating normal form $[13 ; 15]$. It is known that for an $n$-braid $\beta$, whether $1<_{D} \beta$ holds or not is decided in time $O\left(l^{2} \cdot n^{2}\right)$, where $l$ is the length of the input word $\alpha$ with respect to the standard generators $\left\{\sigma_{i}\right\}$ [15, Theorem $\left.1.3(2)\right]$.

Proposition 2.5 Let $\beta$ be an $n$-braid. Then the Dehornoy floor of $\beta$ is computed in time $O\left(l^{2} \log l \cdot n^{4}\right)$ where $l$ is the length of the input word $\beta$ with respect to the standard generators $\left\{\sigma_{i}\right\}$. 
Proof By regarding the standard generators as the band generators, by Proposition 2.4 we obtain an estimation

$$
[\beta]_{D}<\frac{l+1}{n} .
$$

Thus we can determine the Dehornoy floor by at most $O(\log l)$-times of comparison of $\beta$ with $\Delta^{2 L}$ for $|L|<(l+1) / n$. Since the word length of $\Delta^{2 L}$ is $L n(n-1)$, each comparison can be done in time at most $O\left(l^{2} \cdot n^{4}\right)$. Therefore we conclude that we can calculate the Dehornoy floor in time $O\left(l^{2} \log l \cdot n^{4}\right)$.

Thus, the computation of the Dehornoy floor is rather easy, and done in polynomial time with respect to both the length of the input word $l$ and the number of strands $n$.

Remark 2.6 There exist infinitely many families of left-invariant total orderings of the braid groups which contain the Dehornoy ordering, called Thurston-type ordering (see Dehornoy et al [10] or Short and Weist [21]). One can define the Thurston floor of braids by using Thurston type orderings in the same way. The proof of the estimation results for Dehornoy floor in this section remains valid for Thurston floors as well. Thus, our main results (Theorem 1.2 and Theorem 1.3) can be stated by using Thurston floors instead of using the Dehornoy floor.

\subsection{Dehornoy floor and block-strand diagrams}

In this section we explain the relationships between the Dehornoy floor and the blockstrand diagrams, first observed in [18] in slightly weaker form. This is the way we gain information about geometric properties of braids and connect braid ordering and braid foliation theory.

First we recall the notion of the block-strand diagrams and template moves. See Birman and Menasco [8] for the precise definition. A block-strand diagram is a diagram $\mathcal{D}$ of (closed) braids which consists of braid blocks and strands. Each braid block represents an arbitrary braiding but is not allowed to use all braid strands. The only exception is the destabilization template, which is shown in Figure 1 (a). We say a (closed) braid $\beta$ is carried by $\mathcal{D}$ if one can assign braidings for blocks of $\mathcal{D}$ so that the obtained braid is isotopic to $\beta$.

A template $\mathcal{T}=\left(\mathcal{D}_{+}, \mathcal{D}_{-}\right)$is a pair of block-strand diagrams, assigned with the correspondence of blocks between two block-strand diagrams. A template is used to represent a braid move, which is a move of closed braids representing the same links. Typical examples are the destabilization, exchange move and flype templates, which are shown in Figure 1. 
(a)

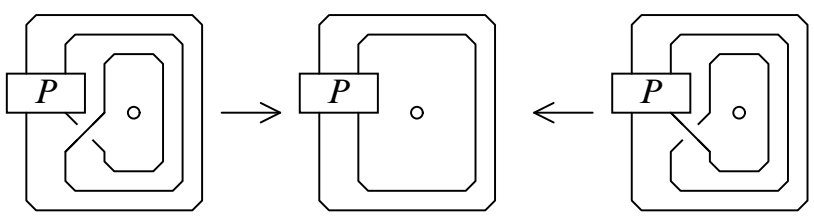

(b)

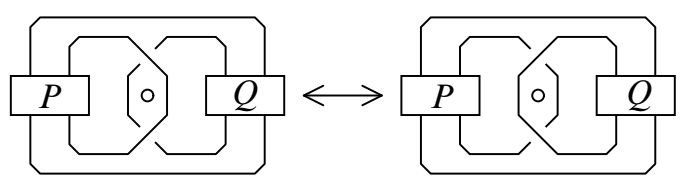

(c)
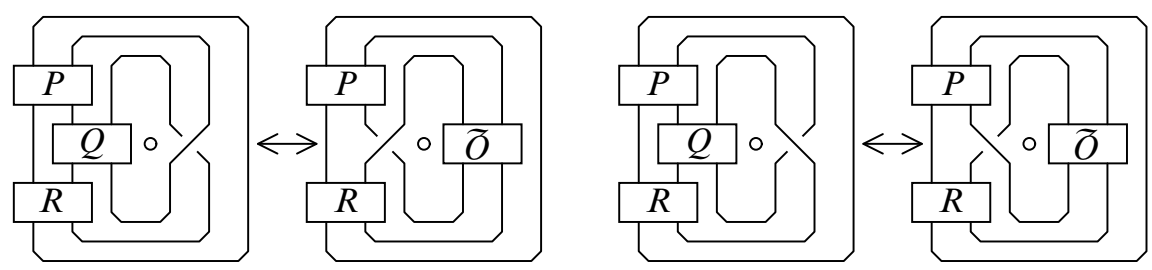

Figure 1: (a) destabilization template (b) exchange move template (c) flype template

In Birman and Menasco's braid foliation theory, which we will review in Section 3, block-strand diagrams have been used to obtain information about closed braids. The key point to use the Dehornoy ordering to study knots is the observation that the Dehornoy floor provides an obstruction for a braid to be carried by a block-strand diagram, as the next Proposition shows. The following Proposition is a generalization of [8, Proposition 1.2.1], which asserts that there exists no block-strand diagram $\mathcal{D}$ that carries all braids.

Proposition 2.7 For a block-strand diagram $\mathcal{D}$, there exists an integer $m(\mathcal{D})$ such that if $[\beta]_{D} \geq m(\mathcal{D})$, then $\beta$ is not carried by $\mathcal{D}$.

Proof A block-strand diagram can be described by a word $V_{1} W_{1} \cdots V_{k} W_{k}$, where $V_{i}$ is braid word carried by a block and $W_{i}$ is a fixed braid word which represents the strands connecting blocks. Since each $V_{i}$ contains not all strands of the braid, we may assume $V_{i}$ contains no $\sigma_{1}^{ \pm 1}$ by modifying the block-strand diagram. Thus we conclude if a braid $\beta$ is carried by $\mathcal{D}$, then $\beta$ is written so that $\beta$ contains at most $m(\mathcal{D}) \sigma_{1}$ or $\sigma_{1}^{-1}$, where $m(\mathcal{D})$ represents the sum of the number of $\sigma_{1}^{ \pm 1}$ in the braid words $W_{1}, \ldots, W_{k}$. By Proposition 2.3, $[\beta]_{\mathcal{D}}<m(\mathcal{D})$.

By using Proposition 2.3 and known results obtained from braid foliation theory, one can prove the following applications of the Dehornoy floor to knot theory. The following theorem is a refinement of the results of Malyutin and Netsvetaev [18]. 
Theorem 2.8 Let $\beta$ be an $n$-braid.

(1) If $\hat{\beta}$ admits a destabilization or an exchange move, then $[\beta]_{D}=0$.

(2) If $\hat{\beta}$ admits a flype, then $[\beta]_{D} \leq 1$.

(3) If $\hat{\beta}$ is a split link, then $[\beta]_{D}=0$.

(4) For each $n$, there exists a positive integer $r(n)$ with the following property: For $\alpha, \beta \in B_{n}$, if $[\alpha]_{D} \geq r(n)$ then the closed braids $\hat{\alpha}$ and $\hat{\beta}$ represent the same oriented link if and only if $\alpha$ and $\beta$ are conjugate.

(5) The minimal value of $r(3)$ is 2 .

Proof Recall that if $\hat{\beta}$ admits a destabilization, then $\hat{\beta}$ also admits an exchange move. If $\hat{\beta}$ admits an exchange move, then $\beta$ is conjugate to a braid $A \sigma_{n-1}^{ \pm 1} B \sigma_{n-1}^{\mp 1}$ where $A, B \in B_{n-1}$. Thus, $\beta$ is conjugate to a braid $\Delta^{-1}\left(A \sigma_{n-1}^{ \pm 1} B \sigma_{n-1}\right) \Delta^{n-1}=A^{\prime} \sigma_{1}^{ \pm 1} B^{\prime} \sigma_{1}^{\mp 1}$ where $A^{\prime}$ and $B^{\prime}$ are $n$-braid words which contain no $\sigma_{1}^{ \pm 1}$. Thus, Proposition 2.3 implies $[\beta]_{D}=0$. The case $\hat{\beta}$ admits a flype is similar.

Assertion (3) follows from the result in [5], which asserts that if $\hat{\beta}$ is a split link, then $\hat{\beta}$ admits an exchange move or $\beta$ is a split braid. Recall that a braid $\beta$ is called a split braid if $\beta$ has a word representative which contains no $\sigma_{i}^{ \pm 1}$ for some $i$. Thus, the Dehornoy floor of a split braid is always zero.

Assertion (4) is a direct consequence of the Markov Theorem Without Stabilization (MTWS) [8] and Proposition 2.7. The MTWS shows, in particular, if two $n$-braids represent the same link, then they are related by the template moves in $\mathcal{T}_{n, n}$, which is a finite set of template moves which sends $n$-braids to $n$-braids.

As for assertion (5), observe that the closure of a 3-braid $\alpha=\sigma_{1} \sigma_{2}^{4} \sigma_{1}^{-1} \sigma_{2}^{6} \sigma_{1}$ admits a flype but $[\alpha]_{D}=1$. Thus, the value 1 in assertion (2) is the best-possible. Now assertion (5) follows from this observation and Birman and Menasco's classification of closed 3-braids [6], which asserts that two closed 3-braids represent the same link if and only if they are conjugate or related by the flype moves.

\section{Braid foliations}

In this section, we summarize the basic machinery of Birman and Menasco's braid foliation theory for an embedded essential surface. For details see Birman and Finkelstein [1] or Birman and Menasco [8].

Fix an oriented unknot $A \subset S^{3}$, called the axis and choose a meridional disc fibration $\left\{H_{\theta} \mid \theta \in[0,2 \pi]\right\}$ of the solid torus $S^{3} \backslash A$. An oriented link $L$ in $S^{3} \backslash A$ is called a 
closed braid with respect to the axis $A$ (and the fibration $\left\{H_{\theta}\right\}$ ) if $L$ intersects every fiber $H_{\theta}$ transversely and each fiber is oriented so that all intersections of $L$ with $H_{\theta}$ are positive.

An embedded closed surface $F$ in the complement of $L$ is essential if $F$ is incompressible and not boundary-parallel. Let $F$ be an essential closed surface in the complement of $L$. Then the intersections of the fibers $\left\{H_{\theta}\right\}$ with $F$ induce a singular foliation of $F$ whose leaves consist of the connected components of the intersection with fibers. The braid foliation techniques are, in short, changing this foliation to obtain a standard and simple position of the surfaces and braids.

We say a fiber $H_{\theta}$ is regular if $H_{\theta}$ transverse $F$ and is singular if $H_{\theta}$ is tangent to $F$. Every nonsingular leaf of the induced foliation is either a simple closed curve or an arc both of whose endpoints lie on the axis $A$. We call the former type of regular leaves $c$-circle and the latter type $b$-arc. Each b-arc in a fiber $H_{\theta}$ separates $H_{\theta}$ into two components. We say a b-arc is essential if both of these components are pierced at least once by $L$. For a c-circle in a fiber $H_{\theta}$, the c-circle bounds a disc $D$ in $H_{\theta}$. The c-circle is called essential if $D$ is pierced at least once by $L$. As shown in [1], we can isotope the surface $F$ so that it satisfies the following conditions.

(1) The axis $A$ pierces $F$ transversely in finitely many points.

(2) For each point $v \in A \cap F$, there exists a neighborhood $N_{v}$ of $v$ such that $F \cap N_{v}$ is the radially foliated disc.

(3) All but finitely many fibers $H_{\theta}$ intersect $F$ transversely, and each of the exceptional fibers is tangent to $F$ at exactly one point. Moreover, each point of tangency is a saddle tangency and lies in the interior of $F \cap H_{\theta}$.

(4) Every regular leaf is essential.

(5) Every c-circle is homotopically nontrivial.

The intersection points of $A$ with $F$ are called vertices. The valence of the vertex $p$ is, by definition, the number of singular leaves which pass through $p$. We say a singular point is a bb-singularity if the singular point is derived from two b-arcs. bc-singularities and cc-singularities are defined in a similar way. Each singular point has a foliated neighborhood in $F$ as shown in Figure 2. We call such a neighborhoods $b b$-tiles, bc-annulus and cc-pants respectively.

A braid foliation of $F$ is classified into the following three types according to the leaves in the braid foliation. We say $F$ is tiled if $F$ is foliated entirely b-arcs and circular if $F$ is foliated entirely c-circles. If $F$ is foliated by both b-arcs and c-circles, we say $F$ is mixed. 


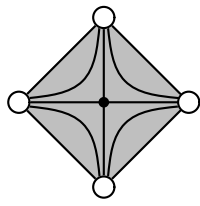

bb-tile

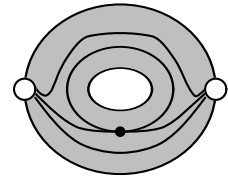

bc-annulus

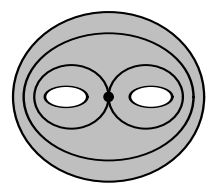

cc-pants

Figure 2: Neighborhoods of singular points

\section{Proof of Theorem 1.2}

Now we begin to prove Theorem 1.2. We prove the Theorem by case-by-case study.

\subsection{Tiled essential surfaces}

First we prove Theorem 1.2 for the tiled case. Throughout this section, we always assume $F$ is tiled.

The tiled surface $F$ is decomposed as a union of bb-tiles. This decomposition defines a cellular decomposition of $F$ which we call a tiling. The notion of vertex and valence we defined in Section 3 coincide with the usual definition of the vertices and valences for this cellular decomposition. First we relate the valence of vertices and the genus of $F$ by using an Euler characteristic argument, which is standard in the braid foliation theory.

Lemma 4.1 (Euler characteristic formula, tiled case) Let $\beta$ be an $n$-braid and $F$ be a tiled essential closed surface in the complement of $\hat{\beta}$. Let $g$ be the genus of $F$ and $V(i)$ be the number of vertices whose valences are $i$. Then, the equality

$$
\sum_{v=1}^{3}(4-v) V(v)+8 g-8=\sum_{v=4}^{\infty}(v-4) V(v)
$$

holds.

Proof Let $E$ and $R$ be the number of 1-cells and 2-cells of the tiling, respectively. Then, $\left(\sum_{v=1}^{\infty} V(v)\right)-E+R=-2 g+2$ holds. On the other hand, each $1-$ cell is the boundary of exactly two 2-cells and each 1 -cell has two vertices as its boundaries, so $2 R=E$ and $\sum_{v=1}^{\infty} v V(v)=2 E$ holds. By combining these equations, we establish the desired equality.

Next we estimate the Dehornoy floor by using a valence of vertex. The following lemma is the core of this paper. 
Lemma 4.2 Let $F$ be a tiled essential closed surface in the complement of the closed braid $L=\widehat{\beta}$, and $g$ be the genus of $F$. If $F$ contains a valence $v$ vertex, then

$$
[\beta]_{D}<\frac{v}{2}
$$

holds.

Proof Let $p$ be a valence $v$ vertex and $\left\{H_{\theta_{1}}, H_{\theta_{2}}, \ldots, H_{\theta_{v}} \mid \theta_{i}<\theta_{i+1}\right\}$ be the sequence of singular fibers which contain the singular leaf passing through the vertex $p$. We denote the b-arc in the fiber $H_{\theta}$ which contains $v$ by $b_{\theta}$. Take a sufficiently small $\varepsilon>0$ so that there are no singular points in each interval $\left[\theta_{i}-\varepsilon, \theta_{i}+\varepsilon\right]$ except in $H_{\theta_{i}}$.

First of all, we isotope the surface $F$ and the closed braid $\widehat{\beta}$ so that each fiber $H_{\theta_{i} \pm \varepsilon}$ is pierced by $L$ at exactly the same points $\left\{p_{1}, p_{2}, \ldots, p_{n}\right\}$ in the fiber $H_{\theta_{i} \pm \varepsilon}=D^{2}$. Then we can decompose the braid $\beta$ as the product of sub-braidings in intervals $\left[\theta_{i}-\varepsilon, \theta_{i}+\varepsilon\right]$ and $\left[\theta_{i}+\varepsilon, \theta_{i+1}-\varepsilon\right]$. We may further isotope $F$ and $\hat{\beta}$ so that the sub-braidings in the intervals $\left[\theta_{i}-\varepsilon, \theta_{i}+\varepsilon\right]$ is trivial. We denote the modified braid by $\beta^{*}=\gamma \beta \gamma^{-1}$.

Then $\beta^{*}$ is decomposed as a product of sub-braidings in intervals $\left[\theta_{i}+\varepsilon, \theta_{i+1}-\varepsilon\right]$. Recall that each b-arc $b_{\theta}$ is essential, that is, $b_{\theta}$ separates a fiber $H_{\theta}$ into two components, both of which are pierced by $\hat{\beta}$. Since b-arc $b_{\theta}$ forms no singularities in each interval $\left[\theta_{i}+\varepsilon, \theta_{i+1}-\varepsilon\right]$, each sub-braiding in $\left[\theta_{i}+\varepsilon, \theta_{i+1}-\varepsilon\right]$ is separated by the surface $F$.

We modify the surface $F$ and $\beta^{*}$ so that we are able to see how the surface $F$ separates each sub-braiding explicitly. This is achieved by modifying the bb-singular points in the standard form, which we define as follows.

The bb-singularity can be regarded as a process of switching two b-arcs $b$ and $b^{\prime}$, along an $\operatorname{arc} d$ connecting $b$ and $b^{\prime}$. As $\theta$ increases, two b-arcs $b$ and $b^{\prime}$ come close along an $\operatorname{arc} d$, then they form the bb-singularity in the singular fiber. After the singularity, the configuration of b-arcs are changed. Thus, the bb-singularity can be described by the sets of $\operatorname{arcs} b, b, d^{\prime}$. Let us consider the most simple configuration of $\operatorname{arcs} b, b^{\prime}, d$ and the bb-singularity as depicted in Figure 3. We call this the standard configuration and the standard bb-singularity.

Let $\beta_{1}$ be the sub-braiding of $\beta^{*}$ in the interval $\left[\theta_{1}+\varepsilon, \theta_{3}-\varepsilon\right]$. We show $\left[\beta_{1}\right]_{D}=0$ by using a method which we call a local modification. We isotope the surface $F$ and the closed braid $\beta$ so that the bb-singularity in $H_{\theta_{2}}$ is the standard bb-singularity by a fiber-preserving isotopy. After such modification, it is easy to obtain the explicit form of the sub-braiding in $\left[\theta_{1}+\varepsilon, \theta_{3}-\varepsilon\right]$. This isotopy is supported in a small neighborhood 


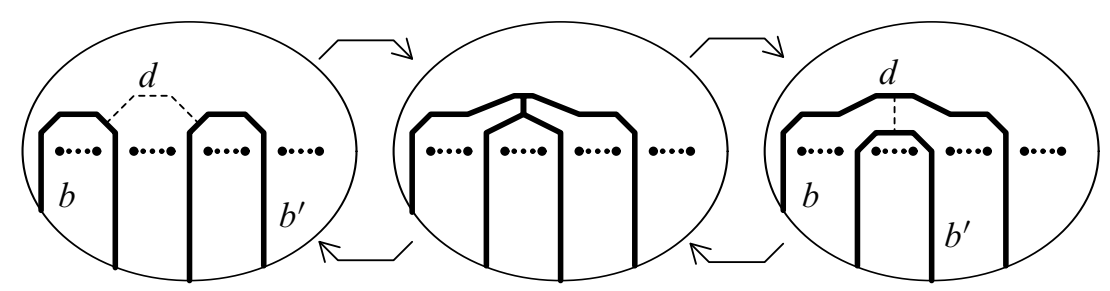

Figure 3: The standard configuration and the standard bb-singularity

of $D^{2} \times\left[\theta_{1}+\varepsilon, \theta_{3}-\varepsilon\right]$. Thus the sub-braiding of the modified braid in the interval $\left[\theta_{1}+\varepsilon, \theta_{3}-\varepsilon\right]$ is conjugate to the original local braid $\beta_{1}$, so we can estimate the Dehornoy floor of $\beta_{1}$ by the modified local braid.

Now we explain the local modification method and desired isotopy of closed braids and surfaces. Let $b=b_{\theta_{2}-\varepsilon}, b^{\prime}$ be the b-arcs in $H_{\theta_{2}-\varepsilon}$ which forms the bb-singularity with $b$ at the singular fiber $H_{\theta_{2}}$, and let $d$ be the arc connecting $b$ and $b^{\prime}$ which describes the bb-singularity in $H_{\theta_{2}}$.

Let $f: D_{n} \rightarrow D_{n}$ be a homeomorphism of $D_{n}$ which sends the configuration of $\operatorname{arcs} b, b^{\prime}, d$ in $H_{\theta_{2}-\varepsilon}$ to the standard configuration. The map $f$ is isotopic to the identity map rel boundary if we regard $f$ as a homeomorphism of the disc $D^{2}$. Let $f_{t}: D^{2} \rightarrow D^{2}$ be an isotopy between the identity map and $f$ as a homeomorphism of $D^{2}$.

Let us consider the isotopy of the tube $\Phi_{t}: D^{2} \times\left[\theta_{1}+\varepsilon, \theta_{3}-\varepsilon\right] \rightarrow D^{2} \times\left[\theta_{1}+\varepsilon, \theta_{3}-\varepsilon\right]$, defined by $\Phi_{t}(x, s)=\left(f_{t}(x), s\right)$. The isotopy $\Phi_{t}$ is extended as a global isotopy $\widetilde{\Phi}_{t}: S^{3} \rightarrow S^{3}$, which is also an isotopy of the closed braid. Moreover, we may assume that the support of the extended isotopy $\widetilde{\Phi}_{t}$ is $D^{2} \times\left[\theta_{1}+\varepsilon / 2, \theta_{3}-\varepsilon / 2\right]$.

Observe that for the modified closed braid $\widetilde{\Phi}_{1}(\hat{\beta})$ and the modified surface $\widetilde{\Phi}_{1}(F)$, the bb-singular point in $H_{\theta_{2}}$ is in the standard form. Let $\beta_{1}^{\prime}$ be the sub-braiding of the modified braid $\widetilde{\Phi}_{1}(\beta)$ in the interval $\left[\theta_{1}+\varepsilon, \theta_{3}-\varepsilon\right]$ and $\gamma_{1}$ be an $n$-braid represented by the homomorphism $f$. Since the isotopy $\widetilde{\Phi}_{t}$ preserves each fiber, $\beta_{1}=\gamma_{1} \beta_{1}^{\prime} \gamma_{1}^{-1}$ holds.

Now we study the braid $\beta_{1}^{\prime}$. Since we have modified the bb-singularity so that it is the standard form, we know the configuration of $b_{\theta}$. In the interval $\left[\theta_{1}+\varepsilon, \theta_{2}-\varepsilon\right]$, b-arc $b_{\theta}$ separates the braiding into the left side and the right side. Similarly, in the interval $\left[\theta_{2}+\varepsilon, \theta_{3}-\varepsilon\right], \mathrm{b}$-arc $b_{\theta}$ separates the braiding into the right side and the left side. Thus, we conclude that $\beta_{1}^{\prime}$ is carried by the block-strand diagram shown in Figure 4 (left). Such a block-strand diagram is modified so that it contains only 


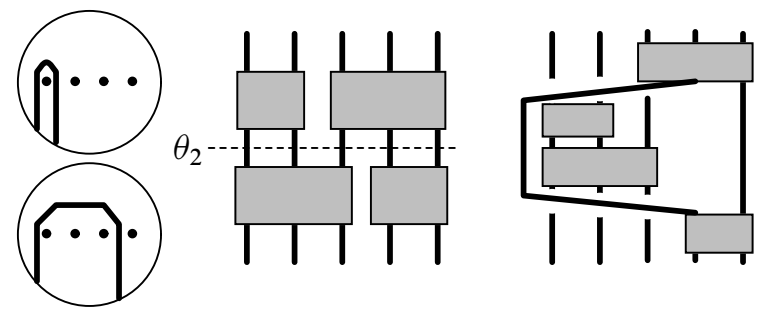

Figure 4: Local braid $\beta_{1}^{\prime}$

one $\sigma_{1}$ and $\sigma_{1}^{-1}$ as shown in Figure 4 (right), hence $\left[\beta_{1}^{\prime}\right]_{D}=0$. Since we observed that $\beta_{1}=\gamma_{1} \beta_{1}^{\prime} \gamma^{-1}$, by Proposition 2.3 we conclude $\left[\beta_{1}\right]_{D}=0$.

See Figure 5 for an example of a local modification of the bb-singularity. Let us consider the bb-singularity described in the upper row of the Figure 5. The configuration of b-arcs $b, b^{\prime}$ and connecting arc $d$ which describe the bb-singularity is mapped to the standard configuration by the homeomorphism $\sigma_{1}^{-1} \sigma_{3}$. Thus, such a bb-singularity is simplified locally by taking a conjugation by $\sigma_{1}^{-1} \sigma_{3}$.

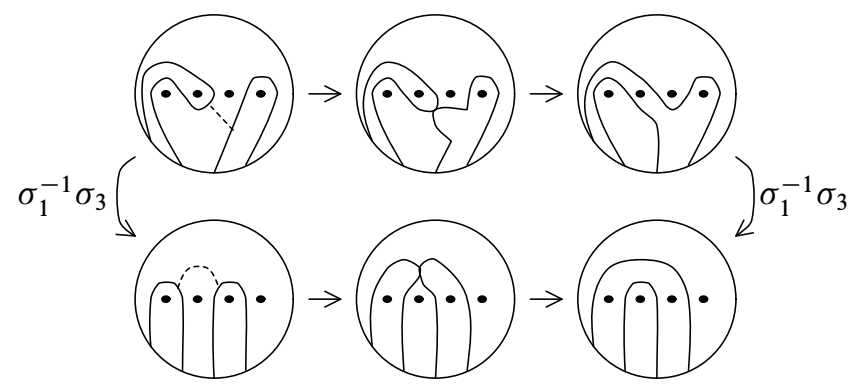

Figure 5: Example of local modification of the bb-singularity

Now we are ready to complete the proof. Let $\lceil v / 2\rceil$ be the minimal integer which is bigger than or equal to $v / 2$ and $\beta_{i}$ be the sub-braiding of $\beta^{*}$ in the interval $\left[\theta_{2 i-1}+\varepsilon, \theta_{2 i+1}-\varepsilon\right]$. By applying the local modification method for each singular point in $H_{2 i}$, we conclude that $\beta_{i}$ is written as a conjugate of the braid $\beta_{i}^{\prime}$ which contains only one $\sigma_{1}$ and $\sigma_{1}^{-1}$. Thus, $\beta_{i}=\gamma_{i} \beta_{i}^{\prime} \gamma_{i}^{-1}$ holds for some braid $\gamma_{i}$. Therefore $\beta$ is written as a product of $\lceil v / 2\rceil$ braids

$$
\begin{aligned}
\beta & =\gamma\left(\beta_{1} \beta_{2} \cdots \beta_{v / 2}\right) \gamma^{-1} \\
& =\left(\gamma \gamma_{1} \beta_{1}^{\prime} \gamma_{1}^{-1} \gamma^{-1}\right) \cdots\left(\gamma \gamma_{\lceil v / 2\rceil} \beta_{\lceil v / 2\rceil}^{\prime} \gamma_{\lceil v / 2\rceil}^{-1} \gamma^{-1}\right) .
\end{aligned}
$$

As each $\beta_{i}^{\prime}$ contains at most one $\sigma_{1}$ and $\sigma_{1}^{-1},\left[\gamma^{-1} \gamma_{1}^{-1} \beta_{1}^{\prime} \gamma_{1} \gamma\right]_{D}=0$ by Proposition 2.3. Thus Proposition 2.2 (3) concludes $[\beta]_{D}<v / 2$. 
Remark 4.3 (Alternative proof of Lemma 4.2) The referee suggested a more braidfoliation theoretic alternative proof of Lemma 4.2. The geometric idea behind the proof is the same, but this approach provides a bit stronger result and make it clearer how the standard braid foliation machinery is used in the proof of Lemma 4.2. We outline the argument here. The author wishes to thank the anonymous referee.

This argument uses more braid foliation theory than we prepared in Section 3. See Birman and Finkelstein [1] for the basics of the braid foliation theory which we did not prepare. The key idea is, we introduce a new braid $\alpha$ which lie on the surface $F$ so that we can see explicitly how the surface $F$ separates the (closed) braid $\beta$ by seeing the braid $\alpha$.

As in the proof of Lemma 4.2, let $p$ be a valence $v$ vertex. First of all, we take a neighborhood $N(p)$ of $p$ on $F$ so that $N(p)$ has the following properties:

(1) $\partial N(p)$ is transverse to the foliation of $F$.

(2) $N(p)$ contains $p$ and all vertices of $F$ which are adjacent to $p$, and contains no other vertices.

(3) $\quad N(p)$ contains all singular points of $F$ which are adjacent to $p$, and contains no other singular points.

Then $N(p)$ is tiled by ab-tiles. Since its boundary $\partial N(p)$ is transverse to the foliation, $\partial N(p)$ is a closed braid with respect to the same axis $A$. We stabilize the closed braid $\partial N(p)$ along an ab-tile of $N(p)$, and obtain a new braid-foliated surface $S$ which is tiled by aa-tiles. Let $\hat{\alpha}$ be the boundary of $S$. See Figure 6 . In this figure, we illustrate an example for the case $v=4$, and $S$ is a disc.
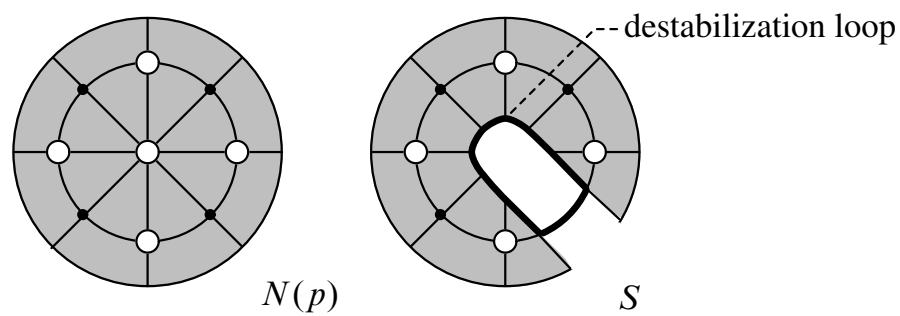

Figure 6: The surface $S$ and the destabilizing loop $l$

From the standard braid foliation theory, we can put the aa-tiled surface $S$ so that the aa-tiling of $S$ induces the disc-band decomposition of $S$. That is, the closed braid $\hat{\alpha}=\partial S$ is written as a product of band generators so that each band generator is boundary of an aa-tile in $S$. See Birman and Hirsch [2] or Ito [14] for details. This is 
achieved by putting $S$ in a good position so that each aa-singularity is in the standard form, like Figure 3.

Now we isotope the surface $F$ and the closed braids $\hat{\alpha}, \hat{\beta}$ so that $S$ has a disc-band decomposition and each aa-tile of $S$ corresponds to a band generator of the braid $\alpha \cup \beta$. This is the crucial step of this proof and the point which corresponds to "making the singular points in the standard form by an local modification" in the proof of Lemma 4.2. The difference is, in this proof we perform a such modification globally by using the aa-tiled surface $S$ and the braid $\alpha$.

Then we can write the braid $\beta \cup \alpha$ as in Figure 7 (right), which has exactly $v$ braid blocks. In Figure 7 (right), dotted line represents the strands of the original braid $\beta$ and usual line represents the strands of the newly-added braid $\alpha$.

Recall that the closed braid $\alpha$ is obtained by stabilizing a closed braid $\partial N(p)$ along an ab-tile. Thus there is the destabilization loop $l$, namely, the bold line in Figure 6 (left). The destabilization loop $l$ bounds a disc, and is not braided with any strands of the braid $\beta$. Therefore we can put the loop $l$ so that $l$ separates each braid block into the right and the left blocks. Thus, the braid $\alpha \cup \beta$ is written as Figure 7 (middle).
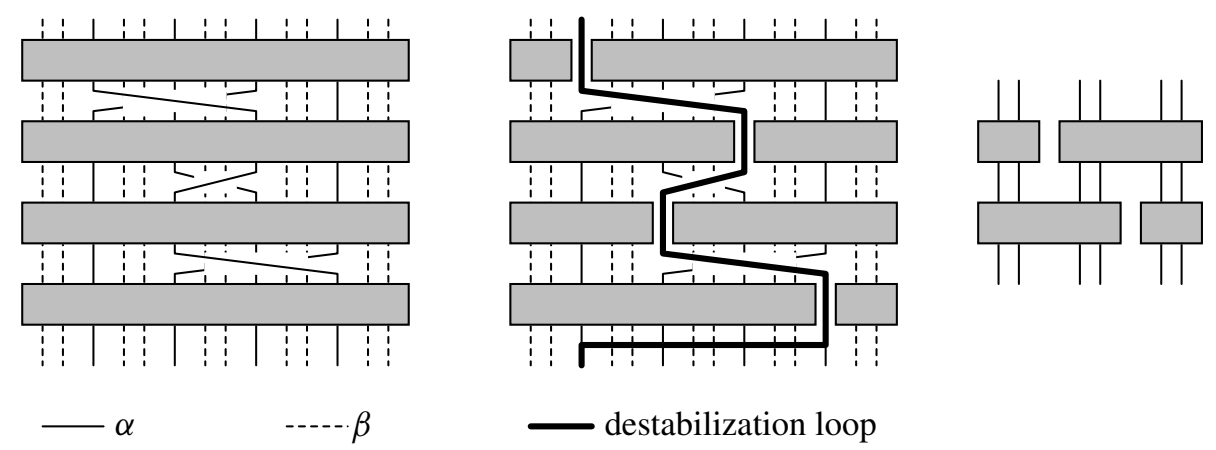

Figure 7: The braid $\widehat{\alpha \cup \beta}$ and the destabilization loop

Now by forgetting the braid $\alpha$, we conclude that the braid $\beta$ is written as a product of the sub-braid which is carried by the block-strand diagram written in Figure 7 (right). As we have shown in Figure 4, such a braid is isotoped so that it contains only one $\sigma_{1}$ and $\sigma_{1}^{-1}$. Since the number of such sub-braidings are at most $\lceil v / 2\rceil$, we obtain the desired result.

Combining Lemmas 4.1 and 4.2, we complete the proof of Theorem 1.2 for tiled surfaces. 
Proof of Theorem 1.2 (1) Let $V(i)$ be the number of valence $i$ vertices. If $V(i)$ is nonzero for some $i \leq 4$, then Lemma 4.2 implies that $[\beta]_{D}<2 \leq g+1$. Therefore we may assume $V(i)=0$ for $i \leq 4$. Then, by Lemma 4.1, we have an equality

$$
8 g-8=\sum_{v=4}^{\infty}(v-4) V(v) .
$$

Since $g$ is not zero, there exist at least two b-arcs in each regular fiber, so $F$ has at least four vertices. Therefore the minimal valence of vertices is at $\operatorname{most} \max \{2 g+2,4\}=$ $2 g+2$. So Lemma 4.2 establishes $[\beta]_{D}<g+1$.

\subsection{Mixed foliated surface}

Next we proceed to the case when $F$ is mixed foliated. The strategy is almost the same, but we need to do some additional arguments to evaluate the valence of vertices by using the genus of $F$. First of all, we observe the same estimation result of the Dehornoy floor.

Lemma 4.4 Let $F$ be a mixed foliated essential closed surface in the complement of closed braid $\hat{\beta}$. If $\beta$ contains a valance $v$ vertex, then the inequality

$$
[\beta]_{D}<\frac{v}{2}
$$

holds.

Proof The proof is almost the same as the proof of Lemma 4.2. The main difference is the existence of bc-singularities. As in the bb-singular point case, we can put a bc-singularity in the standard form by the a local modification method, as we have done in the proof of Lemma 4.2.

Let $p$ be a valence $v$ vertex and $\left\{H_{\theta_{1}}, H_{\theta_{2}}, \ldots, H_{\theta_{v}} \mid \theta_{i}<\theta_{i+1}\right\}$ be the sequence of singular fibers which contain the singular leaves passing the vertex $p$. We denote by $b_{\theta}$ the b-arc in the fiber $H_{\theta}$ which passes the vertex $v$. First of all, we isotope the surface $F$ and the closed braid $\hat{\beta}$ as in the proof of Lemma 4.2, so that we can decompose the braid as the product of sub-braiding.

Let $\beta_{1}$ be the sub-braiding in the interval $\left[\theta_{1}+\varepsilon, \theta_{3}-\varepsilon\right]$. Assume that the singular point in $H_{\theta_{2}}$ is a bc-singularity. Then we perform local modification of bc-singularity as follows.

The bc-singularity is regarded as the amalgamation (or, splitting) of the b-arc and the c-circle. That is, if the bc-singularity is an amalgamation of b-arc and c-circle, then 
bc-singularity is described by the b-arc $b$ and the c-circle $c$, and an arc $d$ connecting $b$ and $c$. The b-arc $b$ and c-circle $c$ approach along the arc $d$, and forms the bc-singularity. After the bc-singularity, the c-circle $c$ is amalgamated to the b-arc $b$. Similarly, if the bc-singularity is a splitting of $\mathbf{b}$-arc, then bc-singularity is described by the $\mathbf{b}$-arc $b$ and an arc $d$. Now let us consider the configuration of $b,(c), d$ and the simplest bc-singularity as shown in Figure 8, and call them the standard configuration and the standard bc-singularity.

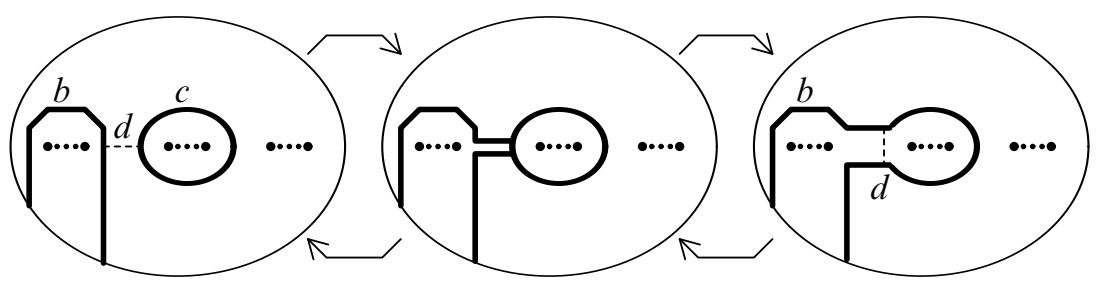

Figure 8: The standard configuration and the standard bc-singularity

Now let $f: D_{n} \rightarrow D_{n}$ be a homeomorphism which sends the configuration $b,(c), d$ in $H_{\theta_{2}-\varepsilon}$ to the standard configuration, and $\widetilde{\Phi}_{t}: S^{3} \rightarrow S^{3}$ be an isotopy constructed from $f$ as in the proof of Lemma 4.2. Then the isotopy $\widetilde{\Phi}_{t}$ puts the bc-singularity in $H_{\theta_{2}}$ into the standard form.

Let $\beta_{1}^{\prime}=\gamma_{1}^{-1} \beta_{1} \gamma_{1}$ be the sub-braiding of the modified braid $\widetilde{\Phi}_{1}(\beta)$ in the interval $\left[\theta_{1}+\varepsilon, \theta_{3}-\varepsilon\right]$. As in the bb-singularity case, by seeing how the $\mathrm{b}$-arc $b_{\theta}$ separates the braid $\beta_{1}^{\prime}$, we conclude the braid $\beta_{1}^{\prime}$ is carried by a block-strand diagram shown in Figure 4 , thus $\beta_{1}^{\prime}$ is written so that it has at most one $\sigma_{1}$ and $\sigma_{1}^{-1}$.

Thus as in the tiled case, we conclude that each local braiding $\beta_{i}$ is conjugate to a braid having only one $\sigma_{1}$ and $\sigma_{1}^{-1}$. Therefore the whole braid $\beta$ can be written as a product of $[v / 2\rceil$ braids having zero Dehornoy floor, so we conclude $[\beta]_{D}<v / 2$.

Next we proceed to an estimation of the minimal valence of the vertices. Since there might be all types of singularities, the decomposition of $F$ into the foliated neighborhoods of singular points dose not define the usual sense of the cellular decomposition of $F$. However, in the normal mixed foliation case which we will define below, one can constructs the cellular decomposition by using the decomposition into regions, as shown in [7].

We say a mixed foliation of $F$ is normal if $F$ has no cc-singularities. In this case, we construct the cellular decomposition of $F$ as follows. First observe that in a normal mixed foliation, each bc-annulus always occurs in pairs, because the c-circle boundary 
of a bc-annulus must be attached to the c-circle boundary of another bc-annulus. Let $W$ be an annulus obtained by attaching two bc-annuli along their c-circle boundaries. Then each component of $\partial W$ has two vertices. By cutting $W$ along the arcs which connect the vertices in different components of $\partial W$ as shown in Figure 9, we obtain two 2-cells. We call such 2-cells be-tiles and the cutting arcs e-edges. Then the decomposition into be-tiles and bb-tiles determines a cellular decomposition of $F$.

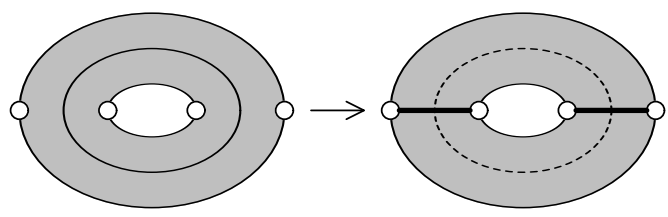

Figure 9: Construction of be-tile

Note that there might exist a be-tile having no singular points or having two singular points, but from the construction of be-tiles, the be-tile without singularities and the be-tile with two singularities must occur in pairs in each neighborhood of vertices. Thus the notion of vertex and valences defined in Section 3 coincide with the usual meaning of vertex and valences in this cellular decomposition. It is easy to see that the same Euler characteristic formula holds for this cellular decomposition.

Lemma 4.5 (Euler characteristic formula, normal mixed case) Let $F$ be a normal mixed foliated surface of genus $g \neq 0$ and $V(v)$ be the number of the valence $v$ vertices. Then,

holds.

$$
\sum_{v=1}^{3}(4-v) V(v)+8 g-8=\sum_{v=4}^{\infty}(v-4) V(v)
$$

Proof A be-tile has exactly four 1-cells as its boundary and each 1-cell is the boundary of exactly two 2-cells, so by the same argument of Lemma 4.1, we obtain the desired result.

Thus, we can estimate the minimal valence of the vertices of normal mixed foliation as in the tiled case. To estimate the valence in general mixed foliated case, we perform a "preimage surgery" to delete cc-singularities.

Let $F$ be a mixed foliated surface and $\mathcal{F}$ be the preimage of $F$. That is, $\mathcal{F}$ is an abstract foliated surface homeomorphic to $F$, forgetting the information of an embedding of $F$ into $S^{3}$. Let $c$ be one of the c-circle boundaries of the cc-pants $P$. This circle $c$ is also 
the boundary of another cc-pants or bc-annulus $Q$. Then we change the two foliated regions $P$ and $Q$ as in Figure 10. Then the modified foliated regions are amalgamated to adjacent foliated neighborhoods so that we can neglect them. Thus, this modification deletes two regions $P$ and $Q$, hence two singularities. We call this operation preimage surgery along $c$. The name "surgery" derived from the fact that ideally we regard this operation as a surgery of the surface $\mathcal{F}$ along the simple closed curve $c$. The preimage surgery is not realizable as a surgery of embedded surface $F$, and the obtained surface is not realized as an embedded surface.
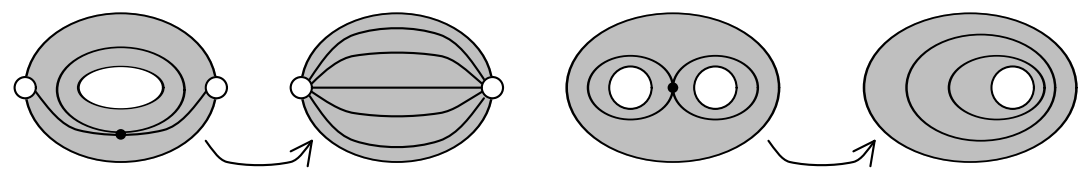

Figure 10: Preimage surgery

From the Euler characteristic reasons, the number of cc-pants is at most $g$. Thus, after $k \leq g$ times of the preimage surgeries we obtain a normal mixed foliated surface $\mathcal{F}^{\prime}$. As we remarked before, $\mathcal{F}^{\prime}$ might not be realized as an embedded surface. However the Euler characteristic formula in Lemma 4.5 remains valid, because the proof of Lemma 4.5 uses only cellular decomposition of $\mathcal{F}^{\prime}$. Therefore we can use Lemma 4.5 for $\mathcal{F}^{\prime}$ to estimate the valence of vertices.

Now we complete the proof of Theorem 1.2.

Proof of Theorem 1.2 (2), (3) Assume $F$ is mixed foliated and after performing a preimage surgery $k$ times, we create an abstract foliated surface $\mathcal{F}^{\prime}$ having no ccsingularities. Let $m$ be the minimal valence of the vertices of $\mathcal{F}^{\prime}$. Now we trace the preimage surgery procedure backwards to estimate the minimal valence of the vertices of $F$. Since one preimage surgery deletes at most one bc-singularity, the original surface $F$ has at most more than $k$ bc-singular points than that of $\mathcal{F}^{\prime}$. Since $F$ is mixed foliated, $F$ has at least one b-arc, so at least two vertices. The singular leaf containing bc-singularity has two vertices as its endpoints, therefore we conclude the minimal valence of the vertices of $F$ is smaller than or equal to $m+k$. Thus, we only need to estimate $m$ to estimate the minimal valence of vertices in $F$.

Let $V(v)$ be the number of the valence $v$ vertices of $\mathcal{F}^{\prime}$. If $V(i)$ is nonzero for some $1 \leq i \leq 3$, then from the argument above, we conclude the minimal valence of vertices of $F$ is smaller than $3+g$, so $[\beta]_{D}<(3+g) / 2 \leq 2 g$. If $k=g$, then $\mathcal{F}^{\prime}$ is a sphere. Then the Euler characteristic formula says that there exist at least one vertex with valance less than 3 , so we obtain the desired result. 
Thus, we assume that $k<g$ and $V(i)$ is zero for $1 \leq i \leq 3$. Then, Lemma 4.5, the Euler characteristic formula for $\mathcal{F}^{\prime}$ provides an equality

$$
8(g-k)-8=\sum_{v=4}^{\infty}(v-4) V(v) .
$$

Since $\mathcal{F}^{\prime}$ has at least two vertices, the minimal valence of the vertices of $\mathcal{F}^{\prime}$ is less than or equal to $\min \{4(g-k), 4\} \leq 4(g-k)$. So from our earlier argument, the minimal valence of the vertices of $F$ is less than or equal to $4(g-k)+k=4 g-3 k$. Now, Lemma 4.4 establishes an inequality $[\beta]_{D}<2 g-3 k / 2 \leq 2 g$.

From statements (1) and (2), we conclude if $[\beta]_{D} \geq 2 g$, then $F$ is neither tiled nor mixed-foliated, hence circular-foliated.

\section{Nielsen-Thurston classification and the geometry of closed braid complements}

In this section we prove Theorem 1.3 and provide some applications.

A satellite knot $K$ represented as a closed braid is called a braided-satellite knot if there exists an essential torus in $S^{3} \backslash K$ which is circular-foliated. In general it is difficult to handle nonbraided satellite knots in braid theory, but fortunately they do not appear under the assumption of Theorem 1.3.

Proposition 5.1 Let $\beta \in B_{n}$ be a braid whose closure $K=\hat{\beta}$ is a nonbraided satellite knot. Then, $[\beta]_{D}<2$.

Proof Assume $[\beta]_{D} \geq 2$. Then Theorem 1.2 implies all essential tori in the complement of $K$ are circular, which contradicts the assumption that $K$ is a nonbraided satellite.

Remark 5.2 In [18], it is proved that if a closure of $\beta$ is a composite knot, then $[\beta]_{D}<2$. Proposition 5.1 can be seen as a generalization of this result because composite knots are special cases of nonbraided satellite knots. The value 2 is the best-possible, because the connected sum of two trefoils is represented as the closure of a 3-braid $\sigma_{1}^{2} \sigma_{2}^{3} \sigma_{1}$, whose Dehornoy floor is 1 .

To treat torus knots, we need the following result due to Menasco. 
Theorem 5.3 (Menasco $[20 ; 19])$ Every torus knot is exchange-reducible. That is, for every closed braid representative $\hat{\beta}$ of the $(p, q)$-torus knot $(p<q)$, there exists a sequence of closed braids

$$
\widehat{\beta}=\widehat{\beta_{0}} \rightarrow \widehat{\beta_{1}} \rightarrow \cdots \rightarrow \widehat{\beta_{m}}=\widehat{\left(\sigma_{1} \cdots \sigma_{p-1}\right)^{q}}
$$

such that each $\widehat{\beta_{i+1}}$ is obtained from $\widehat{\beta_{i}}$ by performing exchange moves, destabilization or isotopy.

Now the proof of Theorem 1.3 is easy.

Proof of Theorem 1.3 Assume that $K=\widehat{\beta}$ is the $(p, q)$-torus knot $(p<q)$. If $n \neq p$, then Theorem 5.3 implies that $K$ admits an exchange move or a destabilization, so we have $[\beta]_{D}=0$ by Theorem $2.8(1)$. This contradicts the assumption that $[\beta]_{D} \geq 2$. If $n=p$, then Theorem 5.3 says $\beta$ is conjugate to $\left(\sigma_{1} \sigma_{2} \cdots \sigma_{p-1}\right)^{q}$, hence $\beta$ is periodic.

To show the converse, recall that periodic braids are conjugate to either $\left(\sigma_{1} \sigma_{2} \cdots \sigma_{n-1}\right)^{S}$ or $\left(\sigma_{1} \sigma_{2} \cdots \sigma_{n-1} \sigma_{1}\right)^{s}$ for some $s$. Since the closure of $\left(\sigma_{1} \sigma_{2} \cdots \sigma_{n-1} \sigma_{1}\right)^{s}$ is never a knot, it is obvious that the closure of a periodic braid is a torus knot.

Next assume $K=\widehat{\beta}$ is a satellite knot. Let $T$ be an essential torus in the complement of $K$. From the assumption $[\beta]_{D} \geq 2$ and Theorem 1.2, $T$ is circular-foliated. Thus, the intersection of $T$ with the fiber $H_{0}$ gives the reducing 1 -submanifolds of $\beta$ as a homeomorphism of $D_{n}$. Hence $\beta$ is reducible. The converse is trivial. Since both classifications are exclusive, we conclude that $\hat{\beta}$ is a hyperbolic knot if and only if $\beta$ is pseudo-Anosov.

As a corollary, we construct infinite, almost disjoint families of hyperbolic knots for each conjugacy classes of pseudo-Anosov element of $\operatorname{MCG}\left(D_{n}\right)$.

Corollary 5.4 Let $[f]$ be a pseudo-Anosov element of the mapping class group of the $n$-punctured disc, and let $\pi: B_{n}=\operatorname{MCG}\left(D_{n}, \partial D_{n}\right) \rightarrow \operatorname{MCG}\left(D_{n}\right)$ be the natural projection. Then the set of closed braids

$$
P([f])=\left\{\hat{\beta} \mid \beta \in \pi^{-1}([f]),[\beta]_{D} \geq 2\right\} .
$$

consists of infinitely many distinct hyperbolic knots. Moreover, for another pseudoAnosov element $[g] \in \operatorname{MCG}\left(D_{n}\right)$, the intersection of $P([f])$ and $P([g])$ are finite unless $[f]$ and $[g]$ are conjugate.

Proof of Corollary 5.4 Since $P([f])$ consists of pseudo-Anosov braids with Dehornoy floor larger than or equal to two, all of their closures are hyperbolic knots 
by Theorem 1.3. Recall Theorem 2.8 (4) says that the closures of $n$-braids having the Dehornoy floor bigger than or equal to $r(n)$ have the unique closed $n$-braid representative up to conjugacy. Therefore $P([f])$ consists of infinitely many distinct hyperbolic knots and $P([f])$ and $P([g])$ have finite intersection unless $[g]$ is conjugate to $[f]$.

Remark 5.5 Using Thurston's theory, one can prove that $P([f])$ contains only finitely many nonhyperbolic knots, without using Theorem 1.3. For a pseudo-Anosov braid $\beta$ and $k \in \mathbb{Z}$, let $L_{k}=\widehat{\Delta^{2 k} \beta}$ and $A$ be the axis of the closed braids $L_{k}$. Then the complements of the link $L_{k} \cup A$ are hyperbolic and homeomorphic for all $k$, because the complement of $L_{k} \cup A$ is the mapping torus of the pseudo-Anosov homeomorphism which represents $\beta$. Since $\Delta^{2}$ corresponds the full-twist along the boundary of $D_{n}$, the complement of $L_{k}$ is obtained from the complement of $L_{0}$ by the Dehn-surgery with the integer coefficient $k$ along the axis $A$. Therefore by Thurston's hyperbolic Dehn surgery theorem, only finitely many of links $\left\{L_{k}\right\}$ can be nonhyperbolic. We also remark that Theorem 1.3 implies that the number of integral surgery along the closed braid axis $A$ which produces a nonhyperbolic knot is at most four.

In some special cases, the situations are much simpler and it is easier to produce hyperbolic knots.

Corollary 5.6 Let $p$ be a prime and $\beta \in B_{p}$ be a nonperiodic braid whose closure is a knot and $[\beta]_{D} \geq 2$. Then $\hat{\beta}$ is a hyperbolic knot.

Proof of Corollary 5.6 From the assumption that $p$ is prime and $\hat{\beta}$ is a knot, the knot $\hat{\beta}$ is not braided satellite. Thus, $\hat{\beta}$ is either a torus knot or a hyperbolic knot, therefore Theorem 1.3 establishes the desired result.

Since to determine given braids are periodic or not is easy, Corollary 5.6 produces very many hyperbolic knots explicitly. For example, under the situation of Corollary 5.6, if the exponent sum of $\beta$ is divisible by neither $p$ nor $p+1$, then the braid $\beta$ is always pseudo-Anosov, hence its closure $\widehat{\beta}$ is a hyperbolic knot if it is a knot.

Finally we provide an example our method efficiently works.

Example 5.7 (Generalized $T$-knots) For $k>0$, let $2 \leq r_{1}<r_{2}<\cdots<r_{k}=n$ be an increasing sequence of integers, and $s_{1}, \ldots, s_{k}$ be arbitrary integers.

A generalized $T$-link $T\left(\left(r_{1}, s_{1}\right), \ldots,\left(r_{k}, s_{k}\right)\right)$ is a link defined as the closure of an $n$-braid

$$
\beta\left(\left(r_{1}, s_{1}\right), \ldots,\left(r_{k}, s_{k}\right)\right)=\left(\sigma_{1} \cdots \sigma_{r_{1}-1}\right)^{s_{1}} \cdots\left(\sigma_{1} \cdots \sigma_{r_{k}-1}\right)^{s_{k}}
$$


This family of links contains an interesting class of links. A generalized $T$-link $L$ is called a $T$-link if all $s_{i}$ are positive. The family of $T$-links is identical with the family of Lorenz links, which are links appearing as a periodic orbit of Lorenz flow [4].

From Theorem 1.3, under the condition either

- $\left|s_{k}\right| \geq 3 r_{k}$ or

- $\left|s_{k}\right| \geq 2 r_{k}$ and $s_{k} s_{k-1}>0$,

the geometric structure of a generalized $T-$ knot $K$ is determined by the NielsenThurston type of the braid $\beta\left(\left(r_{1}, s_{1}\right), \ldots,\left(r_{k}, s_{k}\right)\right)$. In particular, they are noncomposite and not unlink.

\section{References}

[1] J S Birman, E Finkelstein, Studying surfaces via closed braids, J. Knot Theory Ramifications 7 (1998) 267-334 MR1625362

[2] J S Birman, MD Hirsch, A new algorithm for recognizing the unknot, Geom. Topol. 2 (1998) 175-220 MR1658024

[3] J S Birman, K H Ko, S J Lee, A new approach to the word and conjugacy problems in the braid groups, Adv. Math. 139 (1998) 322-353 MR1654165

[4] J S Birman, I Kofman, A new twist on Lorenz links, J. Topol. 2 (2009) 227-248 MR2529294

[5] J S Birman, W W Menasco, Studying links via closed braids. IV. Composite links and split links, Invent. Math. 102 (1990) 115-139 MR1069243

[6] J S Birman, W W Menasco, Studying links via closed braids. III. Classifying links which are closed 3-braids, Pacific J. Math. 161 (1993) 25-113 MR1237139

[7] J S Birman, W W Menasco, Special positions for essential tori in link complements, Topology 33 (1994) 525-556 MR1286930

[8] J S Birman, W W Menasco, Stabilization in the braid groups. I. MTWS, Geom. Topol. 10 (2006) 413-540 MR2224463

[9] P Dehornoy, Braid groups and left distributive operations, Trans. Amer. Math. Soc. 345 (1994) 115-150 MR1214782

[10] P Dehornoy, I Dynnikov, D Rolfsen, B Wiest, Ordering braids, Math. Surveys and Monogr. 148, Amer. Math. Soc. (2008) MR2463428

[11] A Fathi, F Laudenbach, V Poenaru, editors, Travaux de Thurston sur les surfaces, Astérisque 66-67, Soc. Math. France, Paris (1979) MR568308 Séminaire Orsay, With an English summary 
[12] R Fenn, M T Greene, D Rolfsen, C Rourke, B Wiest, Ordering the braid groups, Pacific J. Math. 191 (1999) 49-74 MR1725462

[13] J Fromentin, Every braid admits a short sigma-definite representative, to appear in J. Eur. Math. Soc. arXiv:0811.3902

[14] T Ito, Braid ordering and knot genus, to appear in J. Knot Theory Ramifications arXiv:0805.2042

[15] T Ito, Finite Thurston type orderings on dual braid monoids, to appear in J. Knot Theory Ramifications arXiv:0902.0833

[16] J E Los, Knots, braid index and dynamical type, Topology 33 (1994) 257-270 MR1273785

[17] A V Malyutin, Twist number of (closed) braids, St.Peterburg Math. J. 16 (2005) 791813 MR2106667

[18] A V Malyutin, N Y Netsvetaev, Dehornoy order in the braid group and transformations of closed braids, Algebra i Analiz 15 (2003) 170-187 MR2052167

[19] W W Menasco, An addendum to iterated torus knots arXiv:math.GT/0610566

[20] W W Menasco, On iterated torus knots and transversal knots, Geom. Topol. 5 (2001) 651-682 MR1857523

[21] H Short, B Wiest, Orderings of mapping class groups after Thurston, Enseign. Math. (2) 46 (2000) 279-312 MR1805402

Graduate School of Mathematical Science, University of Tokyo 3-8-1 Komaba, Meguro-ku 153-8914, Japan

tetitoh@ms.u-tokyo.ac.jp, tetitoh@ms.u-tokyo.ac.jp

http://www.ms.u-tokyo.ac.jp/ tetitoh/

Proposed: Joan Birman

Seconded: David Gabai, Shigeyuki Morita
Received: 30 September 2009

Revised: 14 December 2010 